\title{
Combined rigid and flexible endoscopy for tumors in the posterior third ventricle
}

\author{
Jonathan Roth, MD, and Shlomi Constantini, MD, MSc \\ Department of Pediatric Neurosurgery, Dana Children's Hospital, Tel Aviv Sourasky Medical Center, Tel Aviv, Israel
}

OBJECT Tumors leading to occlusion of the sylvian aqueduct include those of pineal, thalamic, and tectal origins. These tumors cause obstructive hydrocephalus and thus necessitate a CSF diversion procedure such as an endoscopic third ventriculostomy (ETV), often coupled with an endoscopic biopsy (EBX). Lesions located posterior to the massa intermedia pose a technical challenge, as the use of a rigid endoscope for performing both an ETV and EBX is limited. The authors describe their experience using a combined rigid and flexible endoscopic procedure through a single bur hole for both procedures in patients with posterior third ventricular tumors.

METHODS Since January 2012, patients with posterior third ventricular tumors causing hydrocephalus underwent dual ETV and EBX procedures using the combined rigid-flexible endoscopic technique. Following institutional review board approval, data from clinical, radiological, surgical, and pathological records were retrospectively collected.

RESULTS Six patients 3.5-53 years of age were included. Lesion locations included pineal $(n=3)$, fourth ventricle $(n=$ $1)$, aqueduct $(n=1)$, and tectum $(n=1)$. The ETV and EBX were successful in all cases. Pathologies included pilocytic astrocytoma, pineoblastoma, ependymoma Grade II, germinoma, low-grade glioneural tumor, and atypical choroid plexus papilloma. One patient experienced an immediate postoperative intraventricular hemorrhage necessitating evacuation of the clots and resection of the tumor, eventually leading to the patient's death.

CONCLUSIONS The authors recommend using a combined rigid-flexible endoscope for endoscopic third ventriculostomy and biopsy to approach posterior third ventricular tumors (behind the massa intermedia). This technique overcomes the limitations of using a rigid endoscope by reaching 2 distant regions.

http://thejns.org/doi/abs/10.3171/2014.9.JNS141397

KEY WORDS endoscopy; rigid; flexible; tumor; hydrocephalus; ventriculostomy; biopsy

$\mathrm{T}$ UMORS leading to occlusion of the sylvian aqueduct include those of pineal, thalamic, and tectal origins. These tumors frequently cause obstructive hydrocephalus and thus necessitate a CSF diversion procedure, such as an endoscopic third ventriculostomy (ETV).

The histopathological differential diagnosis of tumors leading to aqueductal occlusion includes germ cell tumors, ependymomas, and astrocytomas. As the treatment paradigm may change according to pathology (and grade), a tumor biopsy is often indicated. Thus, in many cases a dual procedure is recommended: an ETV combined with an endoscopic biopsy (EBX). ${ }^{17}$

The trajectory for an ETV is pointed more anteriorly than the optimal trajectory for an EBX of a posterior third ventricular tumor. ${ }^{17}$ The foramen of Monro limits the tra- jectory both anteriorly (avoiding injury to the fornix) and posteriorly (avoiding injury to the venous angle and genu of the internal capsule). Various techniques have been suggested to combine an ETV with an EBX, including 1) performing 1 bur hole, between both ideal entry points and using a rigid endoscope (Fig. 1A); ${ }^{15,20-22,26}$ 2) performing 2 separate bur holes: one in an "ideal" location for an ETV and the second more anteriorly in an "ideal" location for a biopsy (Fig. 1B); $3,6,13,14,25,27$ and 3) using a flexible endoscope through a single bur hole..$^{2,8-10,20,23}$ The limitations of these options are a compromise for both the ETV and for the EBX when using a single bur hole and rigid endoscope, the need for 2 entry points and trajectories in the second option, and compromised visual quality and technical limitations using only a flexible endoscope. To overcome

ABBREVIATIONS EBX = endoscopic biopsy; ETV = endoscopic third ventriculostomy; IVH = intraventricular hemorrhage.

SUBMITTED June 22, 2014. ACCEPTED September 25, 2014.

INCLUDE WHEN CITING Published online March 27, 2015; DOI: 10.3171/2014.9.JNS141397.

DISCLOSURE The authors report no conflict of interest concerning the materials or methods used in this study or the findings specified in this paper. 
these limitations, several authors have mentioned using both a rigid and a flexible endoscope for performing both procedures (Fig. 1C). $7,12,20,21$

In the current paper, we present our experience using a combined rigid and flexible endoscopic procedure through a single bur hole for achieving a safer and ideal trajectory for an ETV, as well as an effective method for performing the EBX.

\section{Methods}

Between March 2012 and February 2014, 6 patients with obstructive hydrocephalus secondary to lesions causing aqueductal occlusion underwent surgery at the Tel Aviv Medical Center using the combined endoscopic technique. After receiving institutional review board approval, we retrospectively collected data from clinical, radiological, surgical, and pathological records.

\section{Surgical Technique}

Under general anesthesia, patients were positioned supine with their heads fixed in a 3-pin headholder in a mildly flexed position. Neuronavigation was used in most cases (Brainlab). A single 1-cm bur hole was performed in all cases. The location of the bur hole was chosen to enable a trajectory through the foramen of Monro to the planned location on the tuber cinereum. The trajectory was planned as parallel as possible to the basilar artery to decrease the risk of vascular injury.

Following bur hole placement, the dura was coagulated and opened, and a 12-F peel-away sheath was inserted to the ipsilateral ventricle using frameless navigation. Once the ventricle was cannulated, CSF samples were sent for regular tests as well as cytology and tumor markers (alpha fetoprotein and beta human chorionic gonadotropin). A rigid endoscope $(2 \times 4 \mathrm{~mm}$, Oi HandyPro $)$ was then inserted into the lateral ventricle and through the foramen of Monro to the third ventricle. An ETV was performed using a monopolar probe (with or without current as needed), followed by enlargement of the orifice using the endoscope itself, verifying that the Liliequist membrane was open and the prepontine subarachnoid space communicated.

The rigid endoscope was removed, and a flexible endoscope (Neuro-Fiberscope, Storz) was then inserted into the third ventricle through the same peel-away sheath. The scope was then carefully flexed so that the tip looked backward toward the posterior part of the third ventricle. In all cases the tumor was clearly visualized. Tumor biopsies were obtained using cap forceps. Local bleeds were controlled using irrigation and monopolar cauterization. As a routine, all patients underwent external ventricular drainage for 24-48 hours following surgery.

\section{Results}

\section{Patients}

Six patients $3.5-53$ years of age (only 1 child) underwent surgery using the combined flexible and rigid endoscopic technique. All patients presented with hydrocephalus-related symptoms (with elevated intracranial pressure). Lesion locations included pineal $(n=3)$, fourth ventricle $(\mathrm{n}=1)$, aqueduct $(\mathrm{n}=1)$, and tectum $(\mathrm{n}=1)$ (Table 1 and Fig. 2).

\section{Endoscopic Surgery}

Navigation was used in 5 of 6 patients (1 patient had significantly large ventricles and thus we did not use navigation). The ETV was uneventful in all cases. In 1 patient, the tumor (upper fourth ventricle/distal aqueduct) could not be reached by the rigid endoscope and thus a flexible endoscope was passed though the aqueduct. In the remaining 5 patients, the flexible endoscope was planned to be used. Endoscopic biopsy was uneventful in 5 patients but caused a bleed in 1 patient (pineoblastoma), which subsided with irrigation. During surgery, the interthalamic adhesion was observed and spared in 4 patients (the other 2 patients had no interthalamic adhesion). There were no technical difficulties during surgery.

\section{Pathology}

All specimens were diagnostic. Pathologies included pi-

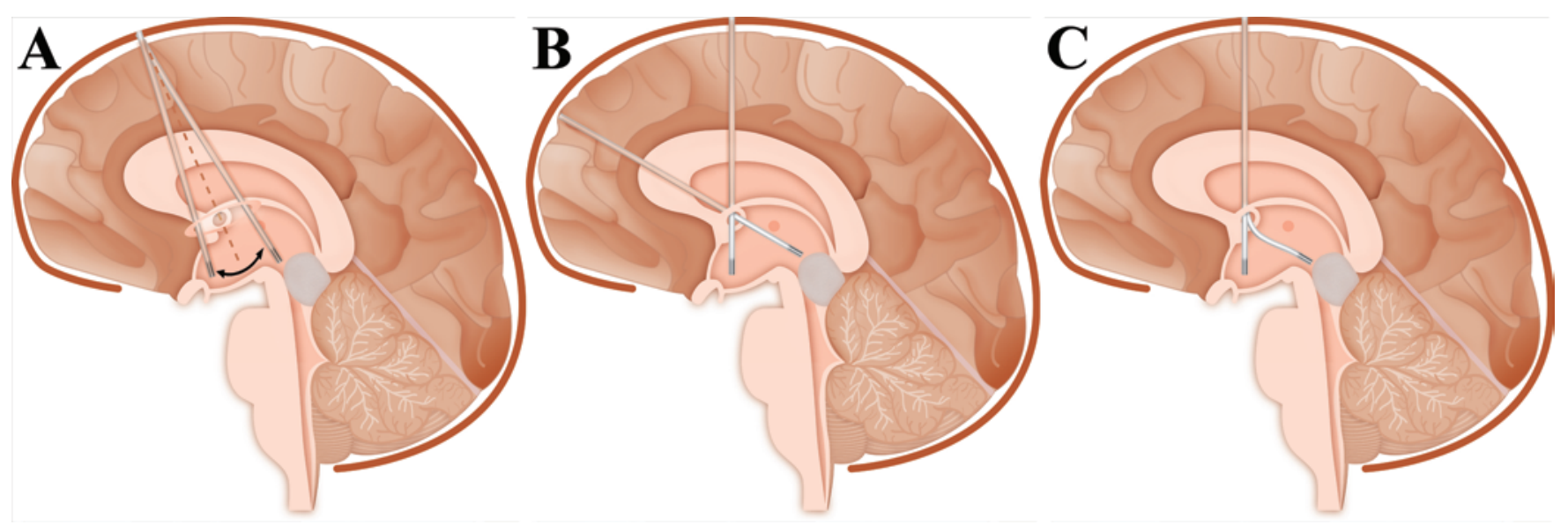

FIG. 1. Illustrations showing the 3 alternatives for combining an ETV and EBX for posterior third ventricular tumors. A: A single compromised entry, with pulling the endoscope anteriorly and posteriorly for the ETV and EBX, respectively. B: Two separate entries and trajectories. C: A single entry and the use of a rigid endoscope for the ETV and a flexible endoscope for the EBX. Copyright Jonathan Roth. Published with permission. Figure is available in color online only. 
TABLE 1. Location and pathological findings in each patient

\begin{tabular}{lll}
\hline $\begin{array}{c}\text { Tumor } \\
\text { Location }\end{array}$ & \multicolumn{1}{c}{ EBX Finding } & \multicolumn{1}{c}{$\begin{array}{c}\text { Final Pathology } \\
\text { (following resection) }\end{array}$} \\
\hline 4th ventricle & JPA & JPA \\
\hline Pineal & Pineoblastoma & Pineoblastoma \\
\hline Pineal & Atypical CPP & \\
\hline Aqueduct & Ependymoma Grade II & Low-grade glioneural tumor \\
\hline Pineal & Germinoma & \\
\hline Tectal & Low-grade glioneural tumor & \\
\hline
\end{tabular}

$\mathrm{CPP}=$ choroid plexus papilloma; JPA = juvenile pilocytic astrocytoma.

locytic astrocytoma, pineoblastoma, ependymoma Grade II, germinoma, low-grade glioneural tumor, and atypical choroid plexus papilloma (Table 1). It should be noted that in the patient with germinoma a blood sample showed alpha fetoprotein and beta human chorionic gonadotropin levels that were within normal range.

Three patients underwent resection of the tumor 1 day to 15 months after the biopsy. The pathology differed from the endoscopic pathology in 1 case (EBX revealed an ependymoma Grade II, whereas resection revealed a lowgrade glioneural tumor).

\section{Complications}

One patient experienced an immediate postoperative intraventricular hemorrhage (IVH) necessitating evacuation of the clots and resection of the tumor (Fig. 2A). This patient had a posterior third ventricular hemorrhagic tumor with an enhancing component involving the massa intermedia (the final pathology was pineoblastoma). After the biopsy procedure, she had a moderate bleed that resolved with irrigation, and clots were endoscopically removed. About 2 hours after surgery, her condition acutely deteriorated due to a massive IVH that necessitated surgical evacuation and removal of the tumor. She never recovered from surgery and died of meningitis following additional CSF drainage procedures. The remaining 5 patients had an uneventful course and did not require a shunt.

\section{Discussion}

Neuroendoscopy is being increasingly used in modern neurosurgery. In the last few years, the scientific community has been struggling to better understand the pathophysiological mechanisms of ventricular pathology, including tumors and hydrocephalus. We also have tried to improve the use of the current available technology. Endoscopes are becoming cheaper and can be connected to many "towers" that are available in hospitals. The combination of endoscopes with open techniques is very interesting. With the maturation of a new technique and keeping the patient's well-being at the center of attention, we can offer a new level of technology and safety. This study focuses on the combined rigid-flexible endoscopic approach for treating patients with hydrocephalus due to tumors causing aqueductal occlusion.

Aqueductal occlusion may arise due to tumors located in the pineal, tectal, aqueductal, posterior third ventricle, and thalamic regions. These deep-seated lesions often present with obstructive hydrocephalus in an acute or semiacute manner. Despite their mass effect (causing aqueductal occlusion), resection of these lesions is not always necessary, such as with pineal germ cell tumors, tectal or
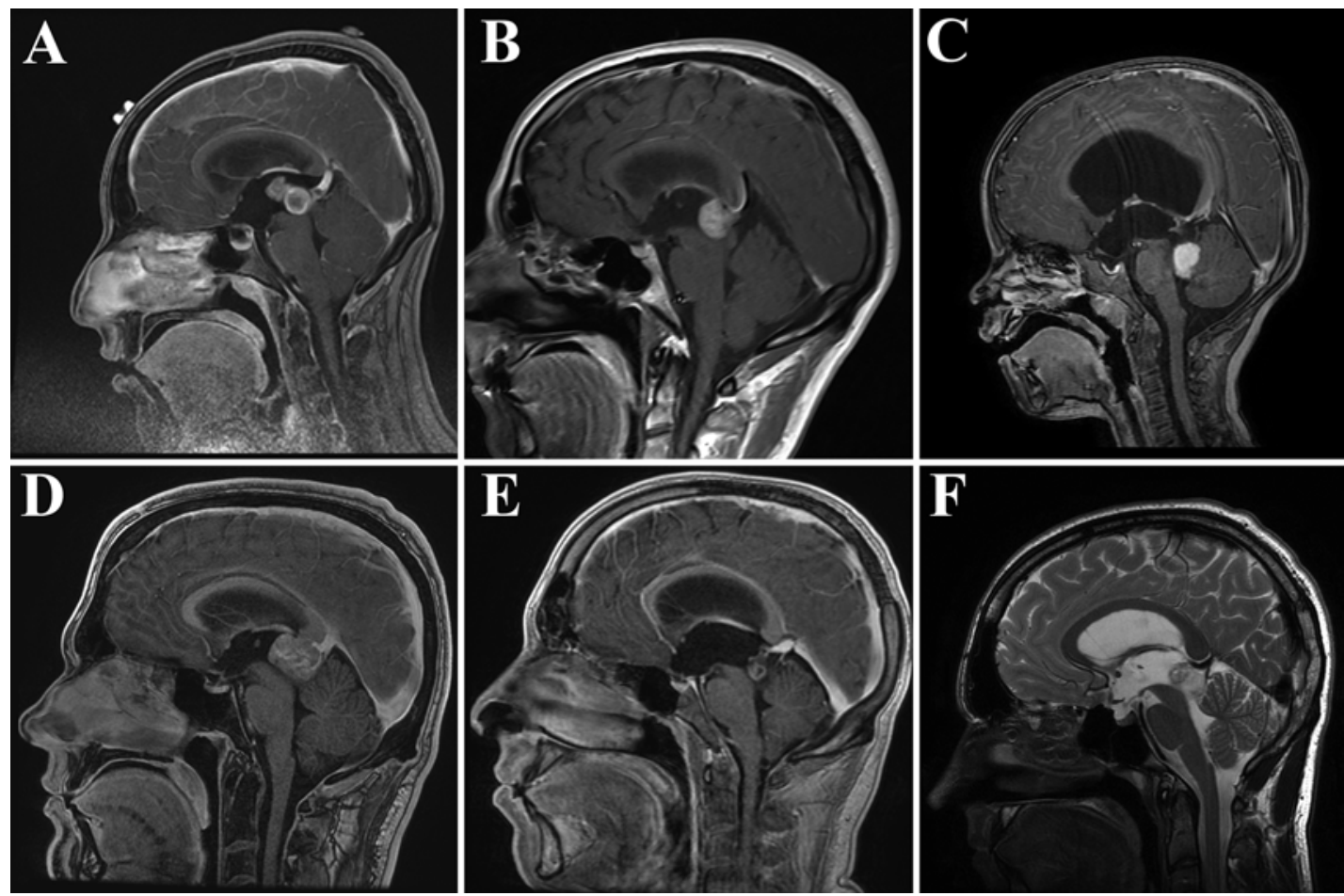

FIG. 2. Magnetic resonance images showing posterior third or fourth ventricular tumors. Note the location of the massa intermedia more anterior to the tumor (B, D, and F). 
aqueductal tumors, and some of the thalamic tumors. Often, the most important treatment is CSF diversion and evaluation of CSF markers and cytological sampling, and obtaining a biopsy of the tumor. Over the last 2 decades, ETV coupled with an EBX has become the mainstream treatment for these lesions.

Performing concurrent ETV and EBX for a posterior third ventricular lesion necessitates 2 separate trajectories, both through the foramen of Monro: an anterior trajectory for the ETV and a posterior trajectory for the EBX. The anterior trajectory is planned more or less parallel to the basilar artery (to avoid vascular injury), while the posterior trajectory enables a clear view and access to the posteriorly located tumor. Often, especially with relatively small and posteriorly located tumors, there is a large angle between the trajectories. This large angle limits the ability to use a single ideal entry for both an ETV and an EBX. To overcome these limitations, various techniques have been applied, each with its pros and cons. One of these techniques is to perform one "compromised" bur hole, midway between the ideal entry sites (Fig. 1A). This compromised entry site enables good access to both targets with a reduced movement relative to the actual entry trajectory. ${ }^{15,20-22,26}$ The compromised location of the bur hole will impose some (but probably of limited clinical importance) pressure on the fornix (when performing the ETV) and on the posterior border of the foramen of Monro (when performing the EBX). In this technique, a rigid endoscope is used. This technique is applicable especially when the tumor is not located extremely posteriorly or when it is larger than about $2 \mathrm{~cm}$. Morgenstern and Souweidane suggested using the massa intermedia as a theoretical border, and they recommended using 2 bur holes for lesions situated behind the massa intermedia and 1 bur hole for lesions extending anterior to the massa intermedia. ${ }^{17}$

A second technique is to perform 2 separate bur holes, each one planned for a separate trajectory (Fig. 1B). The ETV entry is usually at the midpupillary line, about 1-2 $\mathrm{cm}$ anterior to the foramen of Monro, while the EBX entry is placed more anteriorly, at the hairline. Navigation is used to identify the ideal locations of these entry sites. Usually, a rigid endoscope is used for both procedures. Despite several authors recommending this technique, the major drawback is the need for 2 bur holes and 2 separate transcortical passes. $3,6,13,14,25,27$

A third technique is to perform 1 bur hole but use a flexible endoscope..$^{2,8-10,20,23}$ The flexible endoscope may be flexed anteriorly and posteriorly to reach the tuber cinereum or the tumor site and is used to perform both the ETV and the EBX. Another variant of this option was described by Oi et al. in 2000: performing an additional anterior bur hole for applying a rigid endoscope for inspection of the tumor region as well as documenting the presence of "sugar coating" tumor spread, which may not be evident on MRI and may be less visible using a flexible endoscope with relatively inferior optical quality compared with the high-definition rigid endoscopic system. ${ }^{19}$ The main advantage of the flexible endoscope is its ability to reach both distant locations with only 1 entry (and cortical pass). Additionally, it may be possible to reach tumors that are not accessible by a rigid endoscope even when performing a more anterior entry, such as aqueductal tu- mors and tumors in the fourth ventricle. The major limitation of this technique is the reduced visual quality as well as the smaller working channels of the flexible endoscopic systems, which limit the ability to identify tumor spread, as well as potentially affect safety and ease of performing the ETV relative to a rigid endoscope.

A fourth technique, which is described in this paper, is to perform 1 entry hole (located according to the ideal trajectory for an ETV) and to use a rigid endoscope for anatomical inspection, evaluation of tumor spread, and performing an ETV, and to use the flexible endoscope for performing the EBX (Fig. 1C). This technique has been mentioned by other authors as part of larger series in which pineal tumors as well as posterior fossa tumors were treated. However, despite the intuitive combination of endoscopes, this technique is underrepresented in the literature, and these authors have not focused on the qualities of this combination as opposed to the other techniques. ${ }^{7,12,20,21}$

We believe that for posterior third ventricular tumors located posterior to the massa intermedia, especially for small pineal, aqueductal, and tectal tumors (excluding typical tectal gliomas), as well as select cases of fourth ventricular tumors, the flexible endoscope enables easy access to these locations without imposing excessive pressure on the posterior border of the foramen of Monro. Additionally, using the rigid endoscope for the ependymal inspection and for performing the ETV gives the surgeon the best quality instruments for this straightforward procedure. We believe that nowadays, since endoscopic equipment is becoming easily available to many surgeons, a combination of tools should be encouraged given that the patient's safety and ease of procedure are improved.

It is important to note that as opposed to the rigid-flexible endoscopic technique described above (that is, using the rigid endoscope for the ETV and the flexible endoscope for the EBX), a different combined approach may be applied, using the rigid endoscope for performing the EBX, followed by the flexible endoscope for the ETV. The advantages of such an approach are a single entry to the ventricle, better quality endoscope for the EBX, as well as large biopsy specimens and improved equipment availability for the biopsy. The major disadvantages are when there is a large massa intermedia obstructing the view of the rigid endoscope, and in tumors located in the lower part of the aqueduct, or in the fourth ventricle, beyond the reach of a rigid endoscope.

We recommend the routine use of navigation planned according to the goal of the rigid endoscope (either parallel to the basilar artery for an ETV, or toward the tumor if used for the biopsy). This may be spared in cases of significantly large ventricles.

The main limitation of these combined approaches is the need for 2 endoscopic systems. We believe that as endoscopy is being more widely used, it will be more readily accessible. Beyond the instrumentation used for combining ETV and EBX, there is controversy regarding whether to perform the ETV before the EBX, or vice versa. Authors advocating for performing the ETV first state that EBX may lead to a bleed, which will obscure the ability to perform a safe ETV., ${ }^{7,915,21,22}$ As the major (and acute) cause for deterioration is usually hydrocephalus, the pri- 
mary goal of surgery is the ETV, and thus these authors recommend that it should be done first. On the other hand, authors advocating for starting with EBX claim that performing an ETV first will increase the risk for tumor seeding through the ETV to the cranial and spinal subarachnoid space. ${ }^{4,19,23,24}$ Thus, after the EBX they irrigate excessively and only then perform an ETV. Several studies have shown that EBX does not increase the risk for leptomeningeal spread of tumors, and thus we advocate to start with an ETV. ${ }^{11,16,23}$

Another point of dispute is the accuracy of EBX performed using a flexible versus a rigid endoscope. The flexible EBX forceps are smaller than those of rigid systems, affecting the size of the biopsy specimen. Thus, several authors have postulated that specimens obtained with the flexible endoscope are more susceptible to sampling error., ${ }^{1,7}$ On the other hand, others have not demonstrated any reduced rate of diagnosis using the flexible endoscopes. ${ }^{2,10,18,20}$

\section{Safety}

Endoscopic biopsy poses a risk for hemorrhage. In 2 large series of 293 and 714 biopsies reporting on the hemorrhagic rate, the following results were noted. In the first series, moderate to severe IVH occurred in $19 \%$ of patients and $75 \%$ had a mild bleed following the biopsy. ${ }^{5}$ In the second series, $1.4 \%$ had an intratumoral hemorrhage and $2 \%$ had IVH. ${ }^{11}$ The reason for this significant hemorrhagic rate difference may be explained by study population diversity, as well as different surgical techniques and different tumor locations and pathologies. In the current series we experienced 1 moderate bleed following the biopsy. After intraoperative cessation of the hemorrhage, the patient suffered from a significant postoperative hemorrhage, leading eventually to her death. Despite this devastating outcome, diagnostic options are limited in these cases. CSF markers are negative in non-germ cell tumors (such as in this patient, who harbored a pineoblastoma), and stereotactic biopsies entail similar risks and are less controlled, especially in the posterior third ventricle. Using a rigid endoscope enables better instrumental versatility; however, especially in friable tumors, the primary technique for bleeding control is irrigation (as was done and in a successful manner in this case). Using the flexible endoscope enables improved access to a larger tumor region, thus enabling the surgeon to better choose a seemingly safer region for the biopsy. We conclude that in select cases of hemorrhagic tumors, an open biopsy (and possibly a resection) is safer and more controlled than an EBX. In these cases, an ETV should be done first, followed by an open biopsy or resection (after verifying negative markers).

\section{Conclusions}

We recommend using a combined rigid-flexible endoscope for endoscopic third ventriculostomy and biopsy for the treatment of posterior third ventricular tumors (behind the massa intermedia), tumors located in the aqueduct, and tectal tumors (nontypical tectal gliomas) that cause secondary hydrocephalus. We believe that both endoscopes complement each other, enhancing ease of procedure and patient safety.

\section{Acknowledgments}

We thank Mrs. Sigal Friedman for graphic editing and Mrs. Adina Sherer for text editing.

\section{References}

1. Ahn ES, Goumnerova L: Endoscopic biopsy of brain tumors in children: diagnostic success and utility in guiding treatment strategies. J Neurosurg Pediatr 5:255-262, 2010

2. Al-Tamimi YZ, Bhargava D, Surash S, Ramirez RE, Novegno F, Crimmins DW, et al: Endoscopic biopsy during third ventriculostomy in paediatric pineal region tumours. Childs Nerv Syst 24:1323-1326, 2008

3. Boscherini D, Pintucci M, Mazzucchelli L, Renella R, Pesce G: Neuroendoscopic management of a solitary pineal region tumor. Case report of an adenocarcinoma metastasis. Minim Invasive Neurosurg 49:247-250, 2006

4. Chernov MF, Kamikawa S, Yamane F, Ishihara S, Kubo O, Hori T: Neurofiberscopic biopsy of tumors of the pineal region and posterior third ventricle: indications, technique, complications, and results. Neurosurgery 59:267-277, 2006

5. Constantini S, Mohanty A, Zymberg S, Cavalheiro S, Mallucci C, Hellwig D, et al: Safety and diagnostic accuracy of neuroendoscopic biopsies: an international multicenter study. J Neurosurg Pediatr 11:704-709, 2013

6. Costa F, Fornari M, Valla P, Servello D: Symptomatic pineal cyst: case report and review of the literature. Minim Invasive Neurosurg 51:231-233, 2008

7. Depreitere B, Dasi N, Rutka J, Dirks P, Drake J: Endoscopic biopsy for intraventricular tumors in children. J Neurosurg 106 (5 Suppl):340-346, 2007

8. Ellenbogen RG, Moores LE: Endoscopic management of a pineal and suprasellar germinoma with associated hydrocephalus: technical case report. Minim Invasive Neurosurg 40:13-16, 1997

9. Ferrer E, Santamarta D, Garcia-Fructuoso G, Caral L, Rumià $\mathrm{J}$ : Neuroendoscopic management of pineal region tumours. Acta Neurochir (Wien) 139:12-21, 1997

10. Gangemi M, Maiuri F, Colella G, Buonamassa S: Endoscopic surgery for pineal region tumors. Minim Invasive Neurosurg 44:70-73, 2001

11. Hayashi N, Murai H, Ishihara S, Kitamura T, Miki T, Miwa $\mathrm{T}$, et al: Nationwide investigation of the current status of therapeutic neuroendoscopy for ventricular and paraventricular tumors in Japan. J Neurosurg 115:1147-1157, 2011

12. Husain M, Jha D, Vatsal DK, Thaman D, Gupta A, Husain N, et al: Neuro-endoscopic surgery-experience and outcome analysis of 102 consecutive procedures in a busy neurosurgical centre of India. Acta Neurochir (Wien) 145:369-376, 2003

13. Javedan SP, Manwaring K, Smith KA: Treatment of posterior third ventricular central neurocytoma with endoscopic biopsy, endoscopic third ventriculostomy and stereotactic radiosurgery. Minim Invasive Neurosurg 46:165-168, 2003

14. Kim IY, Jung S, Moon KS, Jung TY, Kang SS: Neuronavigation-guided endoscopic surgery for pineal tumors with hydrocephalus. Minim Invasive Neurosurg 47:365-368, 2004

15. Knaus H, Matthias S, Koch A, Thomale UW: Single burr hole endoscopic biopsy with third ventriculostomy-measurements and computer-assisted planning. Childs Nerv Syst 27:1233-1241, 2011

16. Luther N, Stetler WR Jr, Dunkel IJ, Christos PJ, Wellons JC III, Souweidane MM: Subarachnoid dissemination of intraventricular tumors following simultaneous endoscopic biop- 
sy and third ventriculostomy. J Neurosurg Pediatr 5:61-67, 2010

17. Morgenstern PF, Souweidane MM: Pineal region tumors: simultaneous endoscopic third ventriculostomy and tumor biopsy. World Neurosurg 79 (2 Suppl):S18.e9-S18.e13, 2013

18. O'Brien DF, Hayhurst C, Pizer B, Mallucci CL: Outcomes in patients undergoing single-trajectory endoscopic third ventriculostomy and endoscopic biopsy for midline tumors presenting with obstructive hydrocephalus. J Neurosurg 105 (3 Suppl):219-226, 2006

19. Oi S, Shibata M, Tominaga J, Honda Y, Shinoda M, Takei F, et al: Efficacy of neuroendoscopic procedures in minimally invasive preferential management of pineal region tumors: a prospective study. J Neurosurg 93:245-253, 2000

20. Oppido PA, Fiorindi A, Benvenuti L, Cattani F, Cipri S, Gangemi M, et al: Neuroendoscopic biopsy of ventricular tumors: a multicentric experience. Neurosurg Focus 30(4):E2, 2011

21. Pople IK, Athanasiou TC, Sandeman DR, Coakham HB: The role of endoscopic biopsy and third ventriculostomy in the management of pineal region tumours. Br J Neurosurg 15:305-311, 2001

22. Robinson S, Cohen AR: The role of neuroendoscopy in the treatment of pineal region tumors. Surg Neurol 48:360-367, 1997

23. Shono T, Natori Y, Morioka T, Torisu R, Mizoguchi M, Nagata S, et al: Results of a long-term follow-up after neuroendoscopic biopsy procedure and third ventriculostomy in patients with intracranial germinomas. J Neurosurg 107 (3 Suppl):193-198, 2007

24. Song JH, Kong DS, Shin HJ: Feasibility of neuroendoscopic biopsy of pediatric brain tumors. Childs Nerv Syst 26:15931598,2010
25. Veto F, Horváth Z, Dóczi T: Biportal endoscopic management of third ventricle tumors in patients with occlusive hydrocephalus: technical note. Neurosurgery 40:871-877, 1997

26. Wong TT, Chen HH, Liang ML, Yen YS, Chang FC: Neuroendoscopy in the management of pineal tumors. Childs Nerv Syst 27:949-959, 2011

27. Yurtseven T, Erşahin Y, Demirtaş E, Mutluer S: Neuroendoscopic biopsy for intraventricular tumors. Minim Invasive Neurosurg 46:293-299, 2003

\section{Author Contributions}

Conception and design: Roth. Acquisition of data: Roth. Analysis and interpretation of data: Roth. Drafting the article: Roth.

Critically revising the article: both authors. Reviewed submitted version of manuscript: both authors. Approved the final version of the manuscript on behalf of both authors: Roth. Statistical analysis: Roth. Administrative/technical/material support: Roth. Study supervision: Roth.

\section{Supplemental Information \\ Previous Presentation}

Portions of this study were presented orally at the International Federation of NeuroEndoscopy meeting, in Mumbai, December 8-11, 2013.

\section{Correspondence}

Jonathan Roth, Department of Pediatric Neurosurgery, Dana Children's Hospital, Tel Aviv Medical Center, 6 Weizman St., Tel Aviv 64239, Israel. email: jonaroth@gmail.com. 